\title{
Towards Autonomous and Intelligent Systems: The Role of Materials Science and Technology
}

\author{
Tarik Baykara* \\ Mechanical Engineering Department, Dogus University, Istanbul, Turkey \\ *Corresponding author: Baykara T, Mechanical Engineering, MIT Lab, Dogus University, Istanbul, Turkey
}

Submission: January 16, 2019; Published: January 23, 2019

\begin{abstract}
Intelligent/autonomous materials have emerged as a new and innovative concept combining information technologies and materials sciences. In this regard, intelligent/autonomous materials may constitute extensive combination of certain functions such as sensing, actuating and presenting a consistent loop of feedback-feedforward mechanisms. Based on these combinatorial characteristics, the development of intelligent/autonomous materials is important for many scientific, engineering and humanitarian applications such as autonomous robots and vehicles in unpredictable environments i.e., extra-terrestrial operations on the Moon and Mars, real-time monitoring of unreachable zones i.e., deep in caves, devastated zones in an earthquake and other disaster zones. Two different perspectives in this concept are considered starting with the classical scheme of "structureproperty-process- performance" relationship in materials science and technology. However, it is underlined that a new approach is necessary to cover the intelligence and smartness in physical entities through the new perception of "tailoring-modelling-nanostructure and multi-functionality".
\end{abstract}

\section{Introduction}

Autonomous systems, intelligent designs and smart structures are all on the rise and seen with increasing prevalence all over the high-tech world. Design of components, robotics and materials science are the major backbones of these smart, autonomous and intelligent systems. Starting with the extensive range of available materials, critical components are developed by selecting the right materials into a pre-determined shape and form. Finally, robots can be created by integrating all these into one smart and intelligent structure. In this regard, new and innovative materials development along with advanced manufacturing techniques such as additive manufacturing, rapid prototyping, nanotechnology, bioinspired technologies etc., has a key role in these rapidly evolving technologies. Functional materials with the abilities of sensing, controlling, searching, actuation, powering, energy harvesting for the machine intelligence have the utmost importance for the development of robotic systems operating in complex and unpredictable environments. Fortunately, a vast range of materials with the use of combinatorial techniques, composite technologies, nanostructured materials synthesis, and hybrid materials development are now available [1].

Back in mid-1980s, the concept of smart system has emerged as a new generation of high-tech applications. Categorized as passive and active smart materials, they were the new and unique group of advanced materials and they were defined as follows: "Smart materials are the group of materials that change some of the properties (shape, size, colour etc.) when subjected to an external stimulus (magnetic field, pressure, stress, temperature, light, electric field etc.) [2-9]. In this, the smartness was embodied in the sensing, actuation and controlling capabilities. A design of feedback control loop comprised of i. Sensing; ii. Actuation and iii. Control as given in Figure 1 is a typical representation for smart systems.

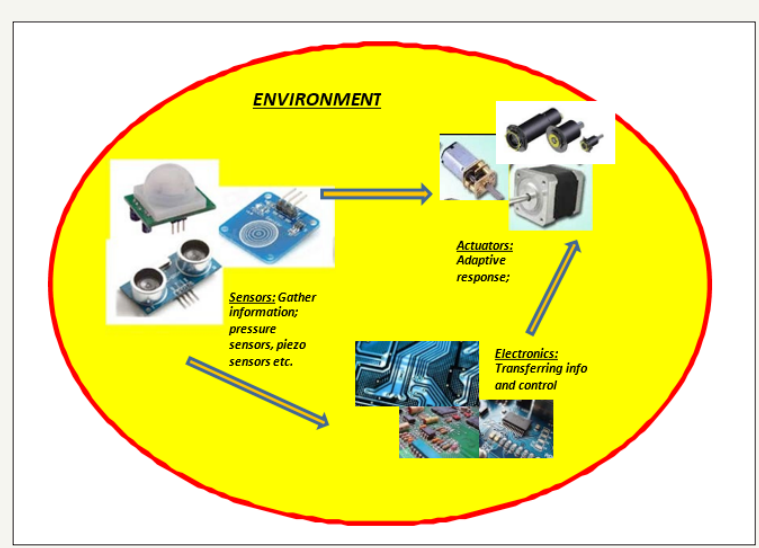

Figure 1: Feedback control loop for a smart system.

i. Sensing; ii. Actuation; iii. Control

The "Smartness" term has gradually evolved into "Autonomous" and "Intelligent" with the advent of the new innovative materials technologies such as follows [6]:

a. Multiferroic materials and composites

b. Biohybrid materials 
c. Phase changing materials (PCMs)

d. Bioinspired/biomimmicked materials

e. Functional and smart materials (piezoelectric, shape memory alloys, etc.)

f. Nanostructures and/or hierarchically built up materials

g. Self-healing materials

These materials exhibit unique and extraordinary properties incorporating smartness, intelligence and autonomy into robust systems which enable them to sense, perceive and detect the external stimuli and change and/or adapt their properties and responses according to the environmental conditions [3]. There is a very wide applications in variety of industrial, defense, health, space and aviation sectors including environmental monitoring, building and construction of smart architectures, aerospace (including the Mars Rover), automotive (including driverless car), medicalsurgical tools and devices (including nanobots in human arterial veins), defense (including UAVs, micro-drones, quad copters etc.), energy (including energy harvesting devices and systems) (Figure 2a-d). Once again, it should be underlined and emphasized that the materials science, engineering and technology are the basis and foundation of these emerging technologies of the future world.

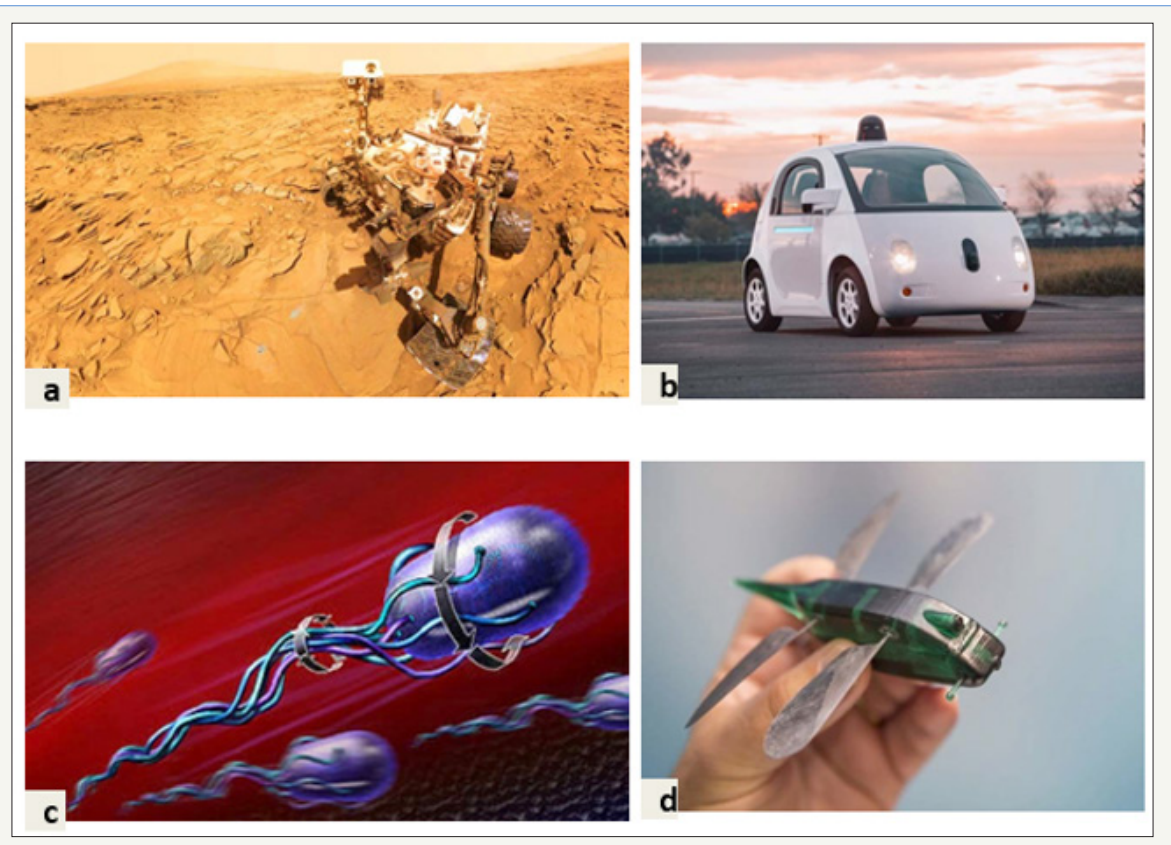

Figure 2:

(a) NASA's Mars Rover, Curiosity (https://www.theatlantic.com/science/archive/2018/09/curiosity-opportunity-nasa-roverproblems $/ 570769 /$ ).

(b) Google Driverless Car, https://www.dezeen.com/2016/02/12/google-self-driving-car-artficial- intelligence-systemrecognised-as-driver-usa/.

(c) A self-propelled nanobot; http://techgenmag.com/2015/01/first-self-propelled-nanobots-successfully-tested-animals/.

(d) UK's micro drone, https://www.theengineer.co.uk/insect-inspiration-uk-defence-drone-mimics-dragonfly-flight/.

\section{Intelligence/smartness through the "structure- property-process- performance" relations}

Materials science and engineering studies during the $20^{\text {th }}$ Century have focused more on the "Structure-Property-Processing and Performance" relationship for a better understanding of material properties based on the fabrication techniques with designed performances as given in Figure 3. This classical depiction is solely for the structural and functional materials which covers limited characteristics [5]. Some of the basic properties were evaluated for further enhancement of the design in applications. As an example, piezoceramics are still a very important group of materials in so many industries such as ultrasound probes/ systems in medical diagnosis, non-destructive testing, underwater communication etc. Piezoelectric property which depends upon the unique crystal structure of certain materials to "sense" pressure and force and responding by generating electrical potential (and surely vice versa) is widely used in many interesting applications.

Based upon such characteristics, other structure-property relations in the following group of materials have played a pioneering role resulting in the "smart systems and smart materials". In such systems, materials with such smart behavior respond various stimuli such as stress, strain, light, electrical field, gas molecules, temperature gradients and pressure etc. The response may have the following characteristics such as movement, changing optical properties, changing elastic modulus, changing surface tension, piezoelectric response, pyro electrical changes etc. These are given in Table 1 that shows the type of smart materials and the smart responses. 
Table 1: The smart materials and smart responses.

\begin{tabular}{|c|c|}
\hline Materials & Smart Response \\
\hline Piezoelectric & Crystals which produces electrical potential when subjected to a stress and vice versa \\
\hline Electrostrictive, Magnetostrictive and Elastorestrictive & The material's changing size in response to an electric and magnetic field and stretching \\
\hline Electrorheological and Magnetorheological & Liquids that can change their viscosity in response to electric field and magnetic field. \\
\hline Thermoresponsive-Shape Memory Alloys & Materials that change shape in response to heat or cold and returning to their original \\
\hline forma and shape.
\end{tabular}

\section{Intelligence/smartness through the "tailoring- multifunctionality-modellingcharacterization" relations}

It is previously pointed out that the relationship given in Figure 3 is far from defining and presenting newly evolving smart materials with limited materials' characteristics such as structure, property, process and performance [5]. New era is strongly demanding complex and dynamic attributes such as innovative tailoring of materials properties and creating new multi-functional materials and rapid deployment, commercialization, extensive collaboration in R\&D \& I (innovation), efficient processing techniques for high quality products [10]. Therefore, a new and emerging relationship within the design of advanced materials engineering is needed and shown in Figure 4; [11].

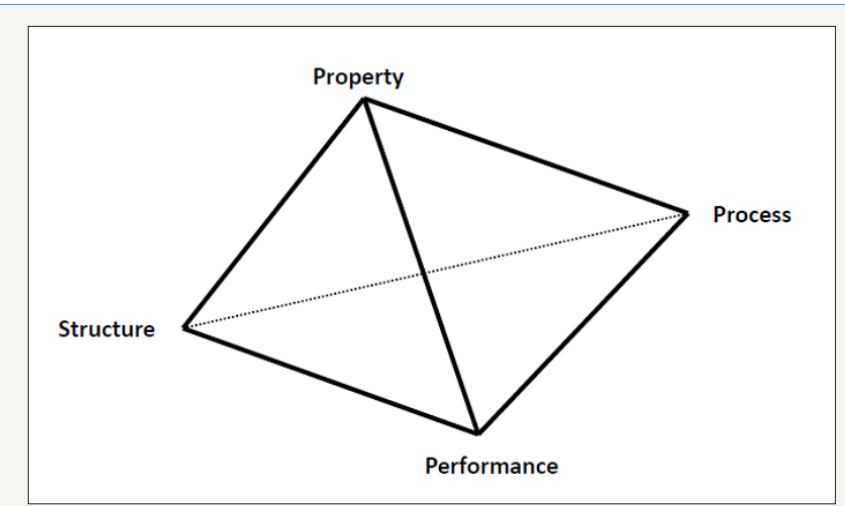

Figure 3: Basic relationship for the materials.

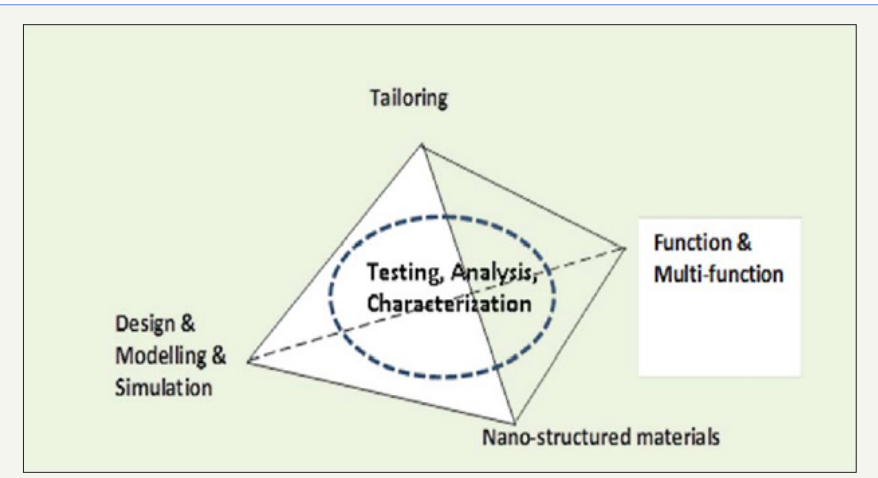

Figure 4: New relationship within the design of novel materials for 21 st century [10].
Newly emerging intelligent/autonomous systems requires materials with certain attributes including sensing, detecting, processing, responding along with data storing and feedback functions. In this respect, intelligent materials should be treated as a new and emerging innovative materials science and technology fields for the upcoming futures. Based upon the nano-structured state on the atomic and molecular scales, these emerging intelligent materials can be tailored to function autonomously and efficiently in an unpredicted and uncontrolled environment. Recent development in additive manufacturing technologies i.e., 3-D printing has been playing an enormous role to build multifunctional systems and helping robotics technologies to advance into a disruptive level of complexity. Intelligent/autonomous materials are the materials with the capabilities of changing and adapting various characteristics in a dynamic and unstable environment and responding accordingly. Following intelligent attributes are typical for these materials and may have extensive applications in so many fields $[5,6,12,13]$ :

a) Self-diagnosis

b) Self-detection

c) Self-learning

d) Self-healing

e) Maintenance

f) Self-corrective and self-controlled functions

g) Recognition and discrimination

h) Sensing, actuation and control

i) Warning, alarming and counter-acting

j) Displaying possible damages, wear and cracks

k) Life cycle prediction and notification

l) Standby ability, termination of operations

m) Reactiveness against unpredicted occurrences

n) Self-replication and cloning (some think as the most extreme condition) 


\section{Intelligent/autonomous applications \\ materials systems}

and

A very diverse group of materials can be treated as potential intelligent/autonomous and/or robotic materials with complex and sophisticated responses when stimulated by an external effect. Therefore, newly emerged group of such materials system is nowadays in the forefront of the scientific and technological challenges. Some of the following materials systems given in Table 2 should be underlined for the prospects of highly intelligent and autonomous systems.

Table 2: Intelligent/autonomous materials [12].

\begin{tabular}{|c|c|}
\hline $\begin{array}{l}\text { Inteligent/Autonomous Mate- } \\
\text { rials }\end{array}$ & $\begin{array}{l}\text { Intelligent/Autonomous Response } \\
\text { and Behaviour }\end{array}$ \\
\hline Self-assembling materials & $\begin{array}{l}\text { Aggregation of structures into the } \\
\text { ordered arrays or patterns that give } \\
\text { function to materials }\end{array}$ \\
\hline Metamaterials & $\begin{array}{l}\text { A metamaterial is defined as an artifi- } \\
\text { cial composite that gains its electrical } \\
\text { properties from its structure rather } \\
\text { than inheriting them directly from } \\
\text { the materials it is composed of [14] }\end{array}$ \\
\hline $\begin{array}{l}\text { Bioinspired and biomimetic } \\
\text { materials }\end{array}$ & $\begin{array}{l}\text { Structural properties and functions } \\
\text { are utilized for further enhancement } \\
\text { via inspired by the natureİ biomimet- } \\
\text { ics are for copying and recreating the } \\
\text { structure-function relations observed } \\
\text { in living entities. }\end{array}$ \\
\hline Energy harvesting materials & $\begin{array}{l}\text { A rapid transport and dissipation of } \\
\text { power using piezo harvesting, solar } \\
\text { and thermoelectric materials. }\end{array}$ \\
\hline Biostable materials & $\begin{array}{l}\text { Biostability is the ability of a material } \\
\text { to present in two stable phases that } \\
\text { can both exist within a given range of } \\
\text { temperatures but above and below } \\
\text { that range only one or the other } \\
\text { phase exists }\end{array}$ \\
\hline $\begin{array}{l}\text { Origami and kirigami inspired } \\
\text { materials }\end{array}$ & $\begin{array}{l}\text { Origami and kirigami, the ancient } \\
\text { techniques for making paper works } \\
\text { of art are being used to develop new } \\
\text { and emerging materials like auxet- } \\
\text { ics-negative Poisson's ratio materials }\end{array}$ \\
\hline Autonomic materials & Autonomic self-healing \\
\hline Computational materials & $\begin{array}{l}\text { Developing materials using direct } \\
\text { computation to the ability to enable } \\
\text { material reconfiguration to achieve } \\
\text { desired autonomous behaviour. }\end{array}$ \\
\hline
\end{tabular}

Some impressive and striking examples of intelligent/ autonomous materials applications are given below:

i. Electrically conductive polypyrrole (PPY) artificial muscles with real time control over a robotic undersea device's speed and direction were developed [12].

ii. Various biomaterials with self-adjustable functions have recently been developed for intelligent operations in medicine such as promoting bone growth, substituting for human skin, liver, kidney, etc. and functioning as a drug delivery system $[5,12,14]$.

iii. Self-healing mechanism illustrated in the integration of two-part epoxy into the bulk polymer coating with the resin phase micro-encapsulated. Upon propagation of a crack, microcapsules are damaged to release the resin and catalyst to fill in the damaged sites recovering approximately $75 \%$ of the original strength [12].

iv. Combining materials, sensing systems and machine intelligence control have come to emerge near-real life like robotics that is called animatronics [13].

\section{Conclusion}

Operations in very complex and sophisticated environments inquire high expectations from systems for fully effective and efficient functions in various areas such as space exploration, disaster management, aviation, automotive, energy, high tech manufacturing etc. In this regard, starting with the smart systems and smart materials back in mid 1980s, newly emerging intelligent/ autonomous materials are becoming extremely important group of materials possessing multifunctional characteristics. Sensing, detecting, processing, responding along with data storing and feedback functions are some of the attributes for these materials. Therefore, a new approach is necessary to treat these materials for further combination with the other technologies such as nanotechnology, biotechnology, mechatronics, control and communication and energy. Using intelligent/autonomous materials which can be tailored to function on robotics, unmanned vehicles (aerial, underwater, land), driverless cars, nano-drones and micro-quadcopters will be the major technologies in near future. It is emphasized that the materials science, engineering and technology is the basis and foundation of these emerging technologies for the future world.

\section{References}

1. David Howard, Agoston EE, Dave Winkler, Danielle FK, Baptiste MJ, et al. (2019) Evolving embodied intelligence from materials to machines. Nature Machine Intelligence 1: 12-19.

2. Matthew NOS, Mahamadou T, Sarhan MM (2017) Smart materials: a primer. International Journal of Advanced Research in Computer Science and Software Engineering 7(3): 43-44.

3. Haddad SS (1994) Intelligent materials-an overview. International Journal of Pressure Vessels and Piping 58(3): 335-344.

4. http://imnr.ro/avanmat/Doc/Workshop/ICPE_CA_Busteni_19.10.06. pdf

5. Toshinori T (1990) A concept of intelligent materials. J of Intelligent Systems and Structures 1: 149.

6. Akhras G (2000) Smart materials and smart systems for the future. Canadian Military Journal.

7. Susmita K (2013) Introduction, classification and applications of smart materials: an overview. American Journal of Applied Sciences 10(8): 876-880.

8. Shawn MW, Michael SS, Samuel C (2019) Stanton in the book-Approaching robotics and autonomous systems as an integrated materials, energy, and control problem. Robotic Systems and Autonomous Platforms, pp. $19-46$. 
9. Tarlk Baykara (2017) A generic transformation of advanced materials science and technologies: towards multi-functional and intelligent multi-materials systems. Research \& Development in Materials Science 1(1): $1-5$.

10. Bhavsar R, Vaidya NY, Ganguly P, Humphreys A, Robisson A, et al. (2008) Intelligence in novel materials. Oilfield Review 20(1): 32.

11. Vonder G, Stillings C, Heiko A (2013) An innovation-focused scenario process-a case from the materials producing industry. Technological Forecasting \& Social Change 80(4): 599-610.
12. https://apps.dtic.mil/dtic/tr/fulltext/u2/a515424.pdf

13. https://repository.asu.edu/attachments/56027/content/Garcia asu_0010N_10048.pdf

14. Mehrdad Mehdizadeh (2015) The impact of fields on materials at microwave and radio frequencies. Microwave/RF Applicators and Probes, pp. 1-33.
Creative Commons Attribution 4.0 International License

For possible submissions Click Here

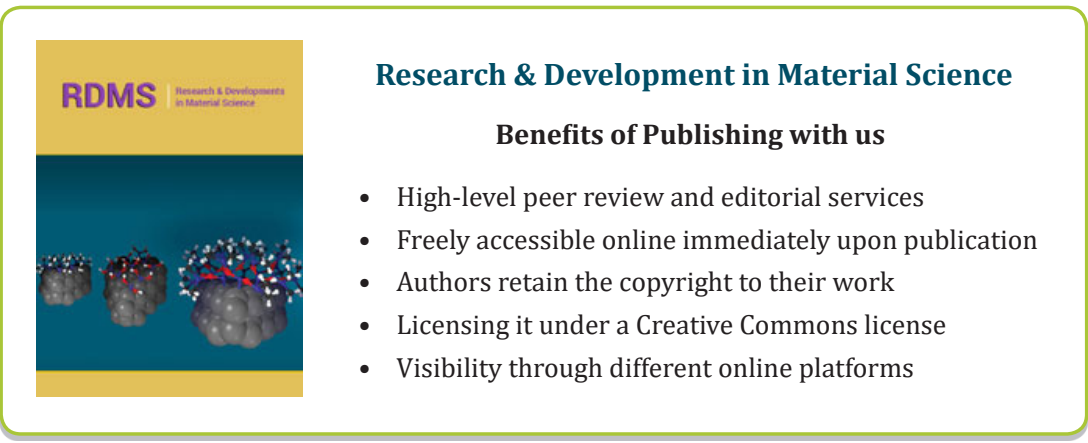

